\title{
Progression of LTE Automatic Neighbour Relations
}

\author{
${ }^{1}$ Gaurav kochar, ${ }^{2}$ Mr. Rakesh Joon (HOD, ECE Deptt.), ${ }^{3}$ Devansh Vats \\ ${ }^{1,2,3}$ Ganga Institute of Technology and Management
}

\begin{abstract}
One of the most time-consuming tasks in today cellular network is the optimization of handover relations. The Automated Neighbor Relations (ANR) feature minimizes the need for the manual configuration of neighbor cell list for the intra and inters frequency handover. ANR in LTE complies with the 3GPP release 8 ANR concept. It is a licensed feature that automatically builds up and maintains a neighbor list used for handover. ANR adds neighbor relations to the cell neighbor list when User Equipment (UE) measurement reports indicate that a possible new neighbour relationship has been found. In this paper, I describe the neighbor relations management and ANR, and effect on ANR in a, commercially deployed network cluster. The results indicate that ANR configures discovered and needed neighbor relations such that handover can be performed in combination with the neighbor relation establishment without dropping the connection and performance in increased by reducing the maintenance of site.
\end{abstract}

Keywords: ANR, 3GPP, LTE, eNodeB, physical cell identity, global unique cell identifier.

\section{Introduction}

The need for even higher data rates, as well as new and Improved services while being mobile have been drivers for the standardization work of the 3G Long Term Evolution [1][2]. The LTE concept consists of an evolved radio access network (E-UTRAN), and an evolved packet core (EPC). It has also been a strong momentum for requirements on management simplicity and cost efficiency of the new system, not the least from the Next Generation Mobile Network (NGMN) association of operators. LTE is based on a rather flat architecture compared to $2 \mathrm{G}$ and $3 \mathrm{G}$ systems see Figure 1 .

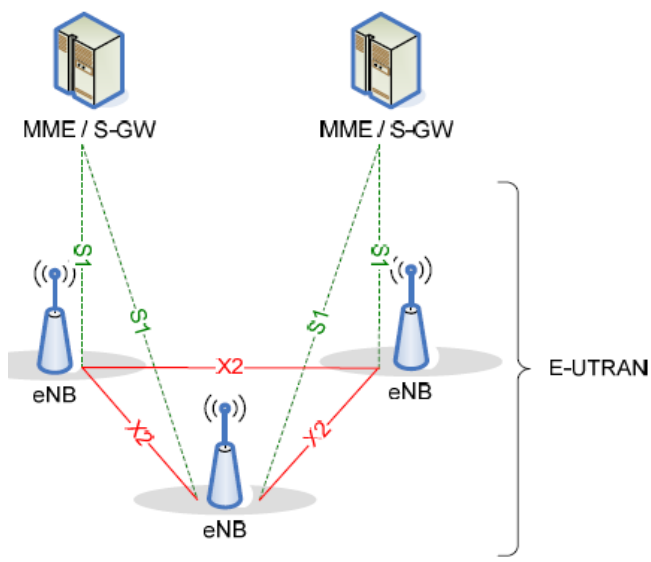

Fig 1(3GPP LTE ANR Architecture)

Each cell is served by an eNodeB or eNB ("base station"), and handovers between cells can be handled either via the Mobility Management Entity (MME) and the S1 interface, or directly between the eNB via the X2 interface. The cell broadcasts an identifying signature or waveform, which can be seen as a "fingerprint", that the mobiles use both as time and frequency reference, as well as to identify cells. Each waveform is enumerated by the Physical Cell Identity (PCI). These identifying signatures are not unique, and can therefore not be used to uniquely identify a neighbor cell In addition; each cell broadcasts as part of the system information a globally unique cell identifier (CGI).

\section{The 3gpp Anr Function}

The ANR function in 3GPP is described [2] and is illustrated by Fig 2. We will discuss NRT management and RRC signalling in more detail in the following subsections.

\section{A. NRT Management:}

Traditionally, the NRT is defined from the operations and maintenance (O\&M) system, and this possibility still exists even when ANR is implemented. However, it is possible to launch base stations without 
any NRT entries at all. Each NRT entry is uniquely identified by a target cell identifier. If the target cell is an LTE cell at the same frequency, this identifier is the CGI, and NRT also contains the PCI. It is the CGI that the eNB uses when signalling to another eNB via the MME, since the MME routes the messages based on eNB identity which is a part of CGI.

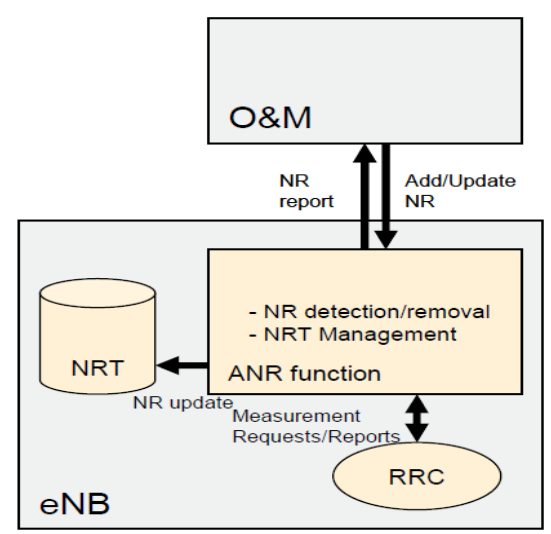

Fig 2(3GPP ANR Function)

The CGI is also used when acquiring via MME the IP address of another eNB, which is used for X2 interface establishment. Additionally, the NRT entry contains information about X2 availability, ANR may remove the neighbor relation or not, and whether the neighbor relation may be used for handover or not.

\section{B. RRC Signalling:}

Two different RRC signalling sequences are discussed in this section - handover when the neighbor relation is established and when not.

The RRC measurement control and report mechanisms can be seen as a toolbox. Typically, the mobile is requested to monitor the observed signal strength of candidate cells relative serving cell signal strength. An alternative is to instead consider the received. Signal quality, but this is not in the 3GPP ANR description. When the relative difference meets a configured criterion, an event-triggered measurement report is signalled to the serving eNB, identifying the target cell by its PCI. Handover is prepared and executed over X2 or S1, informing the serving eNB about access information that the mobile shall use when accessing the target eNB. This mobility control information is conveyed to the mobile, which confirms the handover towards the target eNB.

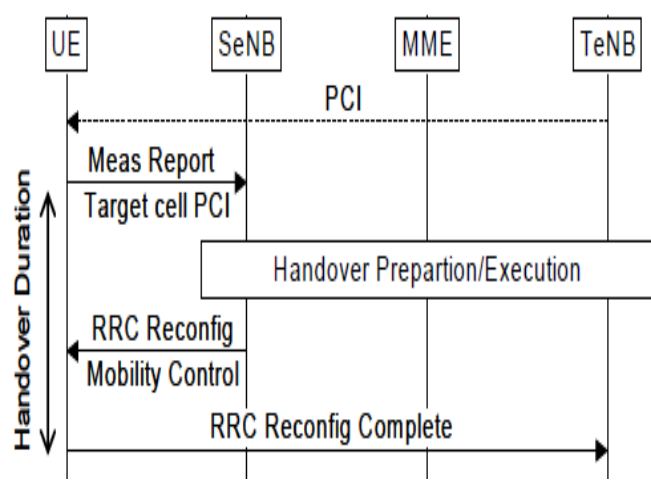

Fig 3(RCC signalling to support handover)

In case the PCI is unknown by the serving cell, the mobile is requested via RRC to decode and report CGI. Additionally, the eNB may define discontinuous transmission cycles during which the mobile is ensured that it does not need to monitor serving cell. These time periods can be used to decode CGI of the target cell. An alternative is that the mobile uses autonomous gaps - essentially neglects any serving cell signalling to create a gap for target cell CGI recovery.

\section{ANR Measurement:}

It is possible to define specific Measurement Objects that specify ANR-specific trigger thresholds and timers for intra-frequency neighbours, i.e. ANR conditions can be more rigorous than conditions for the real handover, 

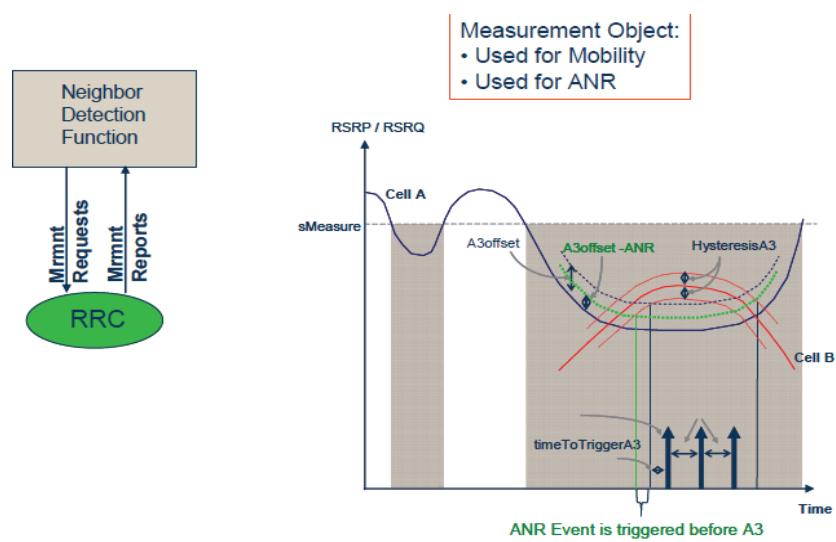

Fig 4. (ANR Measurement)

\section{Related Works}

For an overview on autonomic communication and self optimization,. In $2 \mathrm{G}$ and $3 \mathrm{G}$ systems, the mobiles need NRT information in order to report candidate cells, but in LTE the mobiles can operate without such information. Instead, it is the Radio Access Network that benefit from the NRT. Considering NRT generation, one of early approaches was formulated for GSM, D-AMPS, and PDC. In their approach a set of new test cells (frequencies) are added to the neighbor list of a cell. This enables a mobile to measure cells currently not on the neighbor cell relation list of the cell serving the mobile. Statistics on signal quality (as measured by the mobiles), $\mathrm{HO}$, and drop call rate are used as input to the algorithm.

In WCDMA, the mobiles are capable of detecting and reporting cells not listed in the provided NRT information - detected set reporting (DSR) [4].

Soldani and Ore report results on self-optimization of neighbor cell relation lists for UTRA FDD networks using DSR measurements [3]. The suitability of newly detected neighbours is evaluated using an aggregated performance metric, which includes indicators such as $\mathrm{HO}$ success ratio, $\mathrm{HO}$ share, and Ec/NO. The neighbours are ranked and the best cells are chosen for deployment in the updated NRT. This approach is not directly applicable to LTE, where instead the correspondence to detected set measurements is used in the handover procedure, and that it is possible for the mobile to extract the globally unique cell identifier and report to the eNodeB.

Baliosian and Stadler developed a procedure for creating NRT reported in [3]. Each base station intersects the set of mobiles in its service area with the mobiles in the service area of all other base stations. Neighbor relations are formed if the size of the intersected set is larger than a given threshold $\mathrm{H}$. This algorithm must be executed periodically causing traffic overhead in the network. Appropriate values for the period of the algorithm and the parameter $\mathrm{H}$ must be derived. The latter adds to the list of parameter that need to be set by an operator or developer.

Parodi et al. [6] proposed a method for NRT definition, Where the service area of the cells is approximated and their overlap is computed. Two cells are neighbours if their

Approximated service areas overlap. Antenna and wave propagation models are used to estimate the service areas. The accuracy of the models used highly affects the validity of the generated NRT and, as such, generated NRT lists may be erroneous since antenna and propagation models may be inaccurate due to, e.g., unknown terrain data. In contrast to [6] and [7], the current approach for LTE is relying on mobiles to monitor and report neighbours. This eliminates the need of antenna and propagations models and provides accurate information provided by the mobiles. The ANR function is based on the assumption that the PCIs are locally unique. PCI conflict resolution corresponds to code planning and resolution in WCDMA systems.

\section{A. Intra-LTE(ANR)}

\section{Field Evaluations}

The eNB serving cell A has an ANR function. As a part of the normal call procedure, the eNB instructs each UE to perform measurements on neighbor cells. The eNB may use different policies for instructing the UE to do measurements, and when to report them to the eNB.

1. UE performs measurements on the neighbor cells by measuring their RSRP or RSRQ. Once the thresholds are reached (measurement criteria fulfilled - see Mobility chapter) by decoding PSC and SSC the UE can detect the Physical Cell ID (PCI)

2. The UE sends a measurement report regarding cell B. This report contains Cell B's PCI, but not it's EUTRAN Cell Global Identifier (ECGI). 


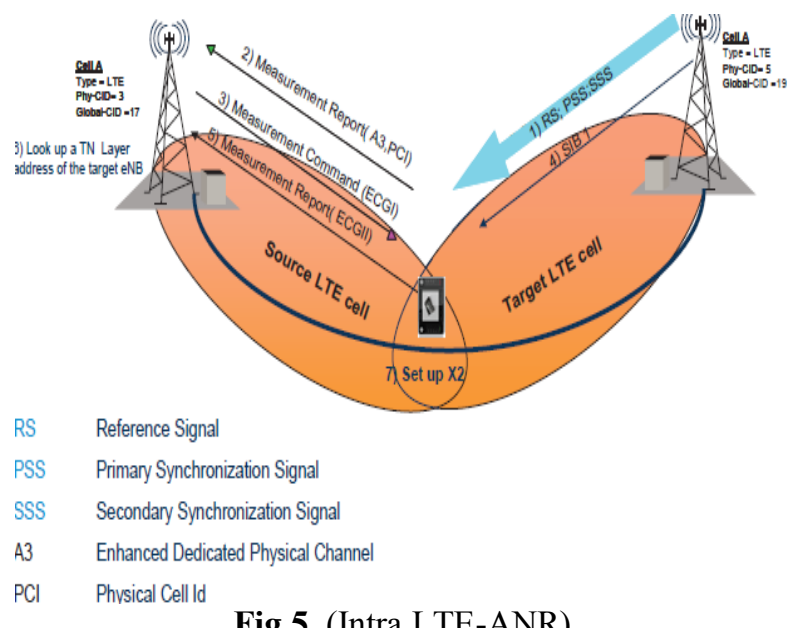

Fig 5. (Intra LTE-ANR)

3. When the eNB receives a UE measurement report containing the PCI, the following sequence may be used.

4. By reading and decoding System Information the UE finds out the new cell's ECGI.

5. The UE reports the detected ECGI (i.e. the PLMN ID, the CSG Indicator and the Cell Identity) to the serving cell eNB.

6. The eNB decides to add this neighbor relation, and can use PCI and ECGI to look up a transport layer address to the new eNB.

\section{B. Inter-LTE(ANR)}

The eNB serving cell A has an ANR function. During connected mode, the eNB can instruct a UE to perform measurements and detect cells on other frequencies.

1. The eNB instructs a UE to look for neighbour cells in the target RATs/frequencies by sending an RRC Reconfiguration Message that contains Measurement Control.

2. The UE reports the PCI of the detected cells in the target RATs/frequencies.

3. The eNB instructs the UE, using the newly discovered PCI as parameter, to read the CGI and the Routing Area Code (RAC) of the detected neighbour cell in the case of GERAN detected cells, CGI, LAC and, RAC in the case of UTRAN detected cells.

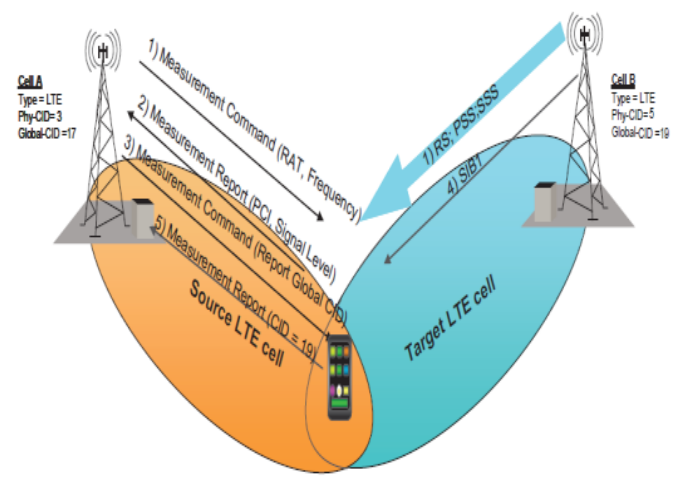

Fig 6. (Inter LTE-ANR)

4. After the UE has read the requested information in the new cell, it reports the detected CGI and RAC (in the case of GERAN detected cells) or CGI, Location Area Code (LAC) and RAC (in the case of UTRAN detected cells) or CGI (in the case of CDMA2000 detected cells) to the serving cell eNB.

5. The eNB updates its inter-RAT/inter-frequency Neighbour Relation Table. In the inter-frequency case the eNB can use the PCI and ECGI for a new X2 interface setup to this eNB.

\section{Conclusions}

In this paper, I describe 3GPP LTE ambitions to support self-organizing networks paradigms for network management. The first feature is automatic neighbor relations, ANR, minimizing costly tuning and configuration work performed in the past. 3GPP ANR mechanisms to configure the neighbor relation tables automatically are described thoroughly. Furthermore, I have present results from intra frequency LTE ANR 
field evaluations in a commercially deployed network cluster in pre-launch state. The results indicate that ANR provides functionality to automatically configure intra-frequency LTE neighbor relations when needed. Moreover, these evaluations indicate that also the Handover of the ANR reporting mobile can be completed successfully. During the trial OSS became just an observation tool, instead of its normal operation - configuration and management.

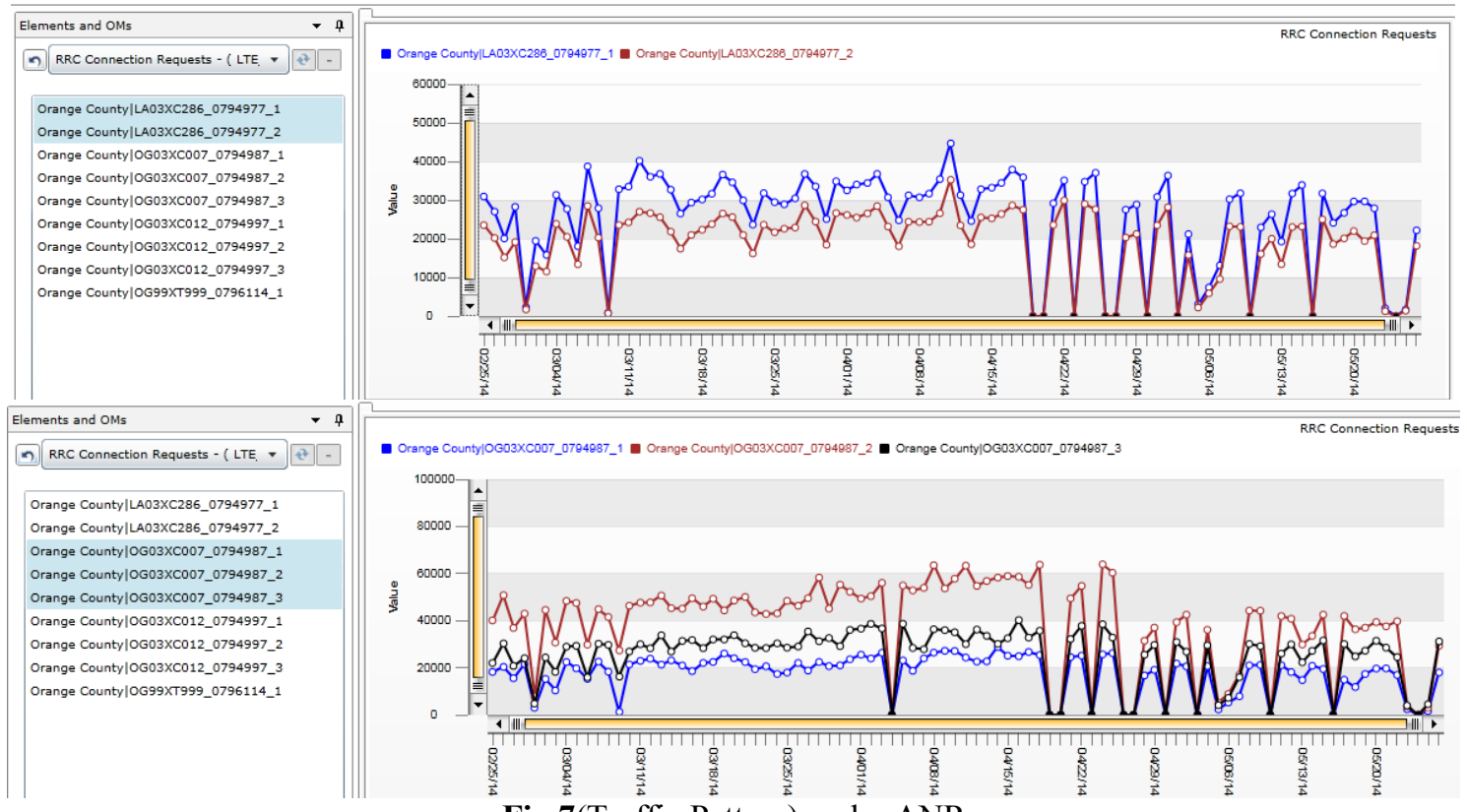

Fig 7(Traffic Pattern) under ANR

\section{References}

[1]. E. Dahlman, S. Parkvall, J. Sköld, and P. Beming, 3G Evolution -HSPA and LTE for Mobile Broadband, Elsevier, 2007.

[2]. 3GPP TS 36.300, v8.12.0, "Evolved Universal Terrestrial Radio Access (E-UTRA) and Evolved Universal Terrestrial Radio Access (E-UTRAN); Overall description"

[3]. LTE L11 "Radio Network Functionality" LND 1087402 R11B.

[4]. 3GPP TS 36.331, "Radio Resource Control (RRC).

[5]. C. Prehofer and C. Bettstetter, "Self-Organization in Communication Networks: Principles and Design Paradigms," IEEE Communications Magazine, July 2005.

[6]. F. Parodi, M. Kylväjä, G. Alford, J. Li, and J. Pradas, "An Automatic Procedure for Neighbor Cell List definition in Cellular Networks," 1st IEEE Workshop on Autonomic Wireless Access (in conjunction with IEEE WoWMoM), Helsinki, Finland, 2007.

[7]. J. Baliosian and R. Stadler, "Decentralized Configuration of Neighbouring Cells for Radio Access Networks," 1st IEEE W orkshop on Autonomic Wireless Access (in conjunction with IEEE WoWMoM), Helsinki, Finland, 2007.

[8]. 3GPP TS 25.133, "Requirements for Support of Radio Resource Management (FDD)."

[9]. Rainer Schoenen Wolfgang ZIrwas, "Coverage analysis of 3GPP-LTE deployment” IEEE Communication Society, 2008. 